\title{
The Support Needs of Nurses Caring for TB Patients at Tshilidzini Hospital in Limpopo Province, South Africa
}

\author{
Hulisani Matakanye*, Augustine Kwame Tugli, Dorah Ursula Ramathuba \\ School of Health Sciences, Department of Public Health, University of Venda, Thohoyandou, South Africa \\ Email: *hmatakanye1@gmail.com
}

How to cite this paper: Matakanye, H., Tugli, A.K. and Ramathuba, D.U. (2019) The Support Needs of Nurses Caring for TB Patients at Tshilidzini Hospital in Limpopo Province, South Africa. Open Access Library Journal, 6: e5862.

https://doi.org/10.4236/oalib.1105862

Received: October 16, 2019

Accepted: November 5, 2019

Published: November 8, 2019

Copyright (ङ 2019 by author(s) and Open Access Library Inc.

This work is licensed under the Creative Commons Attribution International License (CC BY 4.0).

http://creativecommons.org/licenses/by/4.0/

\begin{abstract}
Tuberculosis (TB) is a disease, which is caused by a relatively large, non-motile, rod-shaped pathogen called Mycobacterium tuberculosis. TB is a major cause of illness and death worldwide, especially in Asia and Africa. Despite the fact that TB is a curable illness, the tragedy is that TB remains the biggest killer in the world as a single pathogen. The purpose of this study was to describe the support needs of nurses caring for TB patients at Tshilidzini Hospital in Limpopo Province, South Africa. Qualitative, exploratory and descriptive designs were used. Purposive sampling method was used to select the participants. The personal support needs of six nurses caring for TB patients at Tshilidzini Hospital were explored, and it was guided by data saturation. Data was collected through in-depth individual interviews, field notes were taken and data audio-recorded. Data was analysed using Colaizzi's method of data analysis. Trustworthiness was ensured and ethical considerations were observed. The research findings revealed two major themes from the raw data: support structure available in the hospital and support needs for the nurses. The findings of this study revealed that nurses are in need of special support from the management. Therefore there is an urgent need to address this problem by the Department of Health, Hospital Executive Managers and Nursing Service Manager.
\end{abstract}

\section{Subject Areas}

Nursing

\section{Keywords}

Caring, Needs, Nurses, Patients, Support, Tuberculosis 


\section{Introduction}

Tuberculosis (TB) is a disease, which is caused by a relatively large, non-motile, rod-shaped pathogen called Mycobacterium tuberculosis [1]. TB is a major cause of illness and death worldwide, especially in Asia and Africa. Despite the fact that $\mathrm{TB}$ is a curable illness, the tragedy is that TB poses a grave danger to healthcare workers including nurses because of their exposure to TB patients in health facilities. TB remains the biggest killer in the world as a single pathogen [2].

It is estimated that one-third of the world's population is infected with TB which is responsible for 2 - 3 million deaths annually [2]. A study conducted by Valjee and van Dyk (2014) [3] reported that nurses caring for patients living with TB related illnesses, including HIV/AIDS expressed anxiety, helplessness and vulnerability. Nurses play a major role in caring for TB patients, and those of them in high TB burden settings are at higher risk of developing latent tuberculosis infection (LTBI) when compared to the general populations due to their exposure to the large number of smear-positive TB cases managed at hospitals or healthcare facilities [4]. Working in a high-risk TB setting can lead to harrowing experiences for healthcare workers, especially where health facilities are poorly equipped and managed.

In the Limpopo Province of South Africa, TB is ranked the $4^{\text {th }}$ among the major causes of death [5]. In 2009, the Limpopo Provincial TB Annual Report (2010) [6] stated that the number of TB cases in Vhembe district was 2194, and of these, 750 cases were women of child-bearing age (aged 15 - 44 years). In 2012, Vhembe district was also reported to have the highest number of TB patients in the Limpopo Province [7]. Tshitangano, Maputle and Netshikweta (2013) [8] point out that TB infection control plans are not available at hospitals in Vhembe district and this contributes to the high number of TB patients. Infection control requires both human and material resources. In addition, shortage of space and beds in the wards hinder separation of MDR-TB and TB patients in healthcare facilities and this increases a risk of contracting infection in the wards [9]. In line with this assertion, Gursimrat (2011) [10] argues that one of the major challenges to control $\mathrm{TB}$ infection include poor primary healthcare infrastructure.

According to Tshitangano (2013) [11], Limpopo Province has over 60\% shortage of nurses in its health facilities including Tshilidzini Hospital. In the TB wards of Tshilidzini Hospital, besides managerial duties performed by nurses, they are also involved in providing direct nursing care to the patients. Tshilidzini Hospital is a regional hospital with two TB wards (male and female) consisting of twenty (20) beds. The Hospital has a poor aging infrastructure which is in dire need of refurbishment. In the TB ward, there is congestion and patients have no privacy. Whilst the ward has poor ventilation and sanitation conditions, there are also poor hygiene practices in the wards including regular shortage of water. Above all, there is inadequate supply of personal protective materials such as 
N95 mask. Improper use of protective masks by healthcare workers in TB wards increases the risk of contracting infection. This deplorable condition has been highlighted in an earlier study by Tshitangano, Maputle and Netshikweta (2013) [8] who state that the high risk of TB infection transmission is due to the fact that TB infection control plans are not available at the Hospitals in Vhembe district. It was, therefore, evident that conditions at Tshilidzini Hospital are not conducive for nurses working in the TB wards, hence the researcher wanted to explore and describe the support needs of the nurses working in the TB wards.

\section{Methodology}

\subsection{Study Design}

Qualitative, exploratory and descriptive design was used to explore and describe the support needs of nurses caring for TB patients at Tshilidzini Hospital between 2014 and 2015. The researcher wanted to uncover the support needs of nurses and the meanings that they attached to the events, processes and structures of their lives in the TB wards. Therefore, an exploratory and descriptive design was the most suitable design.

\subsection{Study Site}

This study was conducted at Tshilidzini Hospital in Vhembe District in Limpopo Province, South Africa between 2014 and 2015. Tshilidzini Hospital is a public healthcare facility situated in Thohoyandou, Limpopo Province of South Africa. It is a regional hospital in Vhembe District of Limpopo province with two TB wards and twenty beds. It is a referral hospital for seven community hospitals, such as Louis Trichardt, Elim, Donald Fraser, Musina, Malamulele and Hayani Herberg Hospital. The geographical area is predominated as rural and economic growth depends on agriculture, tourism and mining. According to Wolmarans and Asia (2013) [12], the total population is about 1,294,722, it is comprised of Venda, Tsonga and Sotho speaking people.

\subsection{Study Population}

The study the target population comprised of all nursing staff in all TB wards caring for TB patients at Tshilidzini Hospital in Vhembe District in Limpopo Province at the time of this study. The study included 22 nurses with five years' experience caring for TB patients regardless of their ranks, since the target population was very small.

\subsection{Study Population and Sampling Procedure}

Target population was all nursing staff in all TB wards caring for TB patients at Tshilidzini Hospital in Vhembe District in Limpopo Province at the time of this study. The study included all 22 nurses with five years' experience caring for TB patients regardless of their ranks, since the target population was very small. Purposive sampling was used, to select participants based on their knowledge of 
a phenomenon that was being studied. It was believed that those participants would provide a researcher with the rich data needed to gain insight and discover new meaning in an area of study. Only six nurses were interviewed in this study, even though the researcher envisaged to interview all 22 participants. Interviews were terminated when data saturation was reached, that was when information was repeated and when the participant indicated that there was nothing further that they wanted to say, and no new themes or essences have emerged from the participants. Interviewed participants were four professional nurses and two enrolled nurses. Among the four professional nurses who were interviewed one was a TB focal person.

\subsection{Instrument and Data Collection Procedure}

Data was collected through in-depth individual interviews. Interviews were conducted by the researcher. All six participants signed written consent form before they were interviewed. The interview dates and times were arranged with all participants who were willing to participate in the study prior to data collection date. The interviews were conducted early in the morning as suggested by participants before they could start with their duties to avoid distracting them from their normal wards routine work. Separate interviews with six participants took place in a private office within TB wards, each lasting approximately thirty minutes. All interviews started with an opening question: "What is your support needs when nursing patients who suffer from $T B$ ?"

The research participants freely responded to open-ended questions in narrative form using their own words, thus sharing their own perspectives with the researcher. Questions were not planned in an inflexible manner. The questions were not asked in a prearranged sequence, but the researcher ensured that all relevant topics were covered and that the research focus was kept in mind. The researcher also asked probing questions to guide participants to elaborate further upon their responses where additional information were required or where unclear answers required more clarity. This resulted in gaining in-depth accounts about participants' experiences while caring for TB patients. Data was collected by means of audio-recorded, field notes and in-depth interview. After each interview, the proceedings were transcribed verbatim and entered immediately into an MS word document. The transcripts were then printed for manual analysis.

\subsection{Data Analysis}

Data was analysed using Colaizzi's (1978) [13] methods which comprise the seven steps. Field notes were written down during data collection as participants were talking. Tape-recorded interviews were transcribed verbatim into English. Data was analysed and clustered into themes, categories and sub-categories.

\subsection{Ethical Consideration}

The proposal was submitted and presented to the University Higher Degrees 
Committee (UHDC) of the University of Venda, and recommended for ethical approval by the University Senate and Ethical Committee. Permission to conduct the study was obtained from the Limpopo Provincial Department of Health and Vhembe District Department of Health. Letters were written to the Nursing Service Manager and Chief Executive Officer of Tshilidzini Hospital informing them of the permission granted to conduct the study.

Consent forms were given to every participant to complete. The nature of research was described to the subjects participating in the study and they were informed that they have the right to refuse to participate, or to withdraw from participating if they feel that they cannot continue. The participants were also informed and assured that the information they provided would not be used against them or shared with other people but would be reported as a finding of this study.

Anonymity was also insured in which the respondents did not write down their names or any personality identification. This allowed the participation of respondents to be anonymous. The researcher respected the choices and agreements made with the participants. The initial agreement was not changed without the knowledge of the participants. The participants were not victimized for refusing to participate in the study.

\section{Results}

Two major themes were developed from the findings of this study and sub-themes were also formulated as shown in Table 1 below.

\subsection{Theme 1: Support Structure Available in the Hospital}

Due to the nature of work that nurses provide to the patients it is important that they receive special support from their management and colleagues in order for them to offer quality service. The following sub-themes were discussed under support system available in the hospital: support from the managers, support from the psychologist and appreciation of staff.

\subsubsection{Sub-Theme 1.1: Support from the Managers}

Each hospital aims to deliver good and quality service to the patients. Experienced nursing staff plays a vital role in delivering a highest quality and safest

Table 1. Themes and sub-themes for the support needs of nurses caring for TB patients.

\begin{tabular}{ll}
\hline Themes & Sub-themes \\
\hline $\begin{array}{l}\text { 1. Support structure available in the } \\
\text { hospital }\end{array}$ & $\begin{array}{l}\text { 1.1. Support from the managers } \\
\text { 1.2. Support from the psychologists } \\
\text { 1.3. Appreciation of the staff }\end{array}$ \\
& $\begin{array}{l}\text { 2.1. Good ventilated working environment } \\
\text { 2.2. Good infection control and prevention measures } \\
\text { 2. Support needs for the nurses }\end{array}$ \\
\end{tabular}


care to the patients with the help of the management of the hospital. Nurse managers are widely accepted as the most influential force in staff satisfaction and retention because of their role in work environments. Hospital managements protect and provide staff with the resources to do their job. Within the clinical settings, managers are consistent presence, uniquely positioned with a front-row view of the intricacies of nurse-patient, nurse-physician, and nurseinterdisciplinary team dynamics. Hospitals together with nursing managers are expected to oversee the daily demands of unit operations while developing an environment that fosters nursing excellence and promotes an engaged nursing staff. On the other hand, nursing managers are the vital link between hospital senior executives and direct care nurses. Good management makes hospital to meet its complex target. Below are the comments made by the participants:

One participant indicated that: "We do not receive full support that we need from the managers that we expect in the wards. But we are happy of the fact that the hospital CEO does support us and the nursing service manager give us support, they always come in the wards and walk around with no masks and they understand how we feel in the wards. Our problem is that we do not receive support from other staff, they do not want to come in $T B$ wards since they are scared to contract infection. TB is always associated with HIV/AIDS and we feel stigmatized and people think we have the disease too and they view working in the TB wards as a punishment no one want to interact with us or train as TB nurse."

Another participant said: "Hmmm we believe that for now we just need a special support from the management and all TB program leaders, to give us more knowledge on $T B$, it is crucial that we gain knowledge on how to communicate with those patients and how we nurse them effectively in order to reduce death rate in the wards, spread of diseases in the communities and also those patients who default maybe because they are showing side effects and they have no one to DOT them to continue with treatment."

Another participant indicated that: "I believe that as a nurse it is more crucial that we have meetings with the doctors and other management."

According to the findings of this study it is revealed that even though the support structure from the management is available but there is a need of special support for TB nurses. Lack of support from the management confuses the nursing staff and causes staff to lose interest from their job. The findings of this study concur with the findings of the study that was done by Manyisa and van Aswegen (2017) [14], which revealed that the South African hospital working environment lack resources and managerial support.

\subsubsection{Sub-Theme 1.2: Support from the Psychologist}

Nursing has long been considered one of the most stressful professions. Stress in nursing is attributed largely to the physical labour, suffering and emotional demands of patients and families, long working hours, shift work and interpersonal relationships. Sophisticated healthcare technologies, budget cuts, increas- 
ing workload, and constant organizational changes in some healthcare environments appear to be the factors that have increased stress among nurses. Nurses seem to be overexposed to a range of psychosocial stressors and as a result it is important that they receive Psychological support regularly in order for them to cope in their working environment and remain productive. Below are the comments made by the participants:

Participants indicated that: "The most painful and traumatizing thing in the $T B$ wards is that patients die in large number and it is so stressing to see people dying just before you every day. We really need clinical psychologist to come and give us counseling, but all they do is to schedule an appointment with us and never come to talk to us. We do not get even debriefing sessions as a result we feel that we are losing it, at times we just can't cope."

The findings of this study revealed that nurses do not receive personal psychological support even though the support structure is there in the hospital but their needs are not considered and attended to. A study that was done by Mametja (2013) [15], reported concerns that were raised nurses, that they needed counselling, not only when they have contracted the disease, but in order for them to cope with the demands of the high number of patients who no longer recover from the illness, but die as the result of HIV/AIDS The findings of this study is in agreement with the findings of the study that was done by Arjun (2011) [16], which concludes that nurses caring for MDR-TB patients did not receive psychological support from the clinical psychologists when they need it neither was there a functional employee assistance programme available at the institution.

\subsubsection{Sub-Theme 1.3: Appreciation of Staff}

Hospitalized patients require more than a dozen different daily medications that are administered at various hours and in multiply ways. Although physicians have the responsibilities to diagnose infections and prescribe medications for patients, nurses are responsible for actively caring, feeding, bathing and dispensing medication for hospitalized patients throughout the day. Nurses are in the front line of fighting all kinds of diseases in the world, and they risk their lives to provide quality healthcare service in the hospital. Nurses provide primary care services across a wide spectrum of healthcare settings, their duties range from caring, teaching, counseling and researching more effective ways to deliver care. It is of paramount important that each hospital has a day to appreciate nursing staff for the good work that they are doing of risking their lives to provide care to the patients. Below are the comments made by the participants:

One participant said: "The hospital doesn't even appreciate us for the good work that we are doing by risking our lives in a daily basis. We do not even get a danger allowance in case we contract infection in the wards. Instead we are being told that if we are found with $T B$ we would rather be moved to work in another ward which is not a $T B$ ward. We receive free periodic medical examination and free medication in case we are diagnosed with $T B, h m m m$ and if you 
skip the periodic medical examination and you happen to contract infection you will be told that you did not get the disease in the wards since TB is everywhere."

Another participant indicated that: "We do not receive TB awareness campaign or just a small day where the management just appreciates us of the great work that we are doing. At times I even think of considering other opportunities." Even the families of patients blame us for everything that goes wrong in the patients they do not appreciate the effort we put to care for the patients.

According to the findings of this study it is revealed that TB nurses are less appreciated for what they are doing. TB nurses are at the frontline of the fight against the highly infectious disease that affects many people. They do not receive danger allowance and the hospital does not celebrate TB day as a way of appreciating TB nurses as pioneers in the hospitals who risk their lives in order to fight TB disease. In support of these findings, Loghmani, Borhani and Abbaszadeh (2014) [17], reported that the participants' experiences were that the families thought that nurses did not do their best for the patient and that they abused the patients. The findings of this study concurs with the findings of the study that was done by Sodeify, Vanaki and Mohammadi (2013) [18], which concludes that South African nurses were well supported, appreciated, recognized and well rewarded in other countries since they provide quality service to the patients under very difficult circumstances with limited resources, this is why they leave South Africa to work in other countries.

\subsection{Theme 2: Support Needs for the Nurses}

Evidence indicates that nurses have a heavy workload and they work under pressure on their daily basis. They are expected to have high level of independence, well developed problem solving abilities and leadership responsibilities. Regardless of all the skills they have acquired they still express needs that are not met by the healthcare managements. The following sub-themes were developed under support needs for the nurses: good ventilated working environment, good infection control and prevention measurements and special support from the management.

\subsubsection{Sub-Theme 2.1: Good Ventilated Working Environment}

TB is transmitted through the airborne route. Poorly designed or overcrowded healthcare facilities play an important role of TB transmission and also increase chances of cross-infection between patients' also healthcare workers. HCWs are essential in the fight against TB infection and they should be protected. Good ventilated working environment should have natural, mechanical and negative pressure ventilation to reduce the risk of spreading TB infection in the wards. Natural ventilation relies on open doors and windows to bring fresh air in the wards from outside which dilutes the concentration of particles such as droplet nuclei containing mycobacterium tuberculosis. Negative pressure ventilation prevents contaminated air from the outside to flow into the wards. Mechanical ventilation is used if there is a free flowing air from the outside of the wards. 
Poor ventilated TB wards expose healthcare workers and patients into a high risk of contracting TB infection. Below are the comments made by the participants:

Participants said: "We are working in an old building that needs refurbishment, the infrastructure is very old but anyhow it is not a big problem, our biggest concern is that the $T B$ wards are not designed in a way which is suitable for TB wards, they are different from all other $T B$ wards from other hospitals. The management promised us that they will build us new TB wards that are well ventilated, that have good airflow, fans and regularly serviced UV lights as well as big windows and patients waiting area to allow airflow. The wards will have nursing station and there will be demarcation between TB, MDR-TB and XDR-TB. We really need that wards because we are not safe here."

The findings of this study revealed that nurses expressed the needs of a well-ventilated TB wards in the hospital. The TB wards layout was not suitable and lacked ventilation which increased risk of contagion. The working environment was not safe and did not meet the national standard guideline for TB wards setting as recommended by South Africa (2007) [19]. According to the study that was conducted by Narasimhan, Wood, Maclntyre and Mathai (2013) [20], overcrowding and poorly ventilated environment increase the risk of acquiring TB infection.

\subsubsection{Sub-Theme 2.2: Good Infection Control and Prevention Measures}

Good infection control and prevention in the healthcare settings reduce the risk of contracting TB infection. TB setting should have the following TB infection control and prevention measures; workplace and administrative control measures, environmental control measures and personal control measures. People who work or receive care in healthcare settings are at higher risk of being infected by TB; therefore it is necessary to have a TB infection control plan. Healthcare facilities should design policies and procedures for TB control; it should be reviewed periodically and evaluated for effectiveness to determine the actions necessary to minimize the risk for transmission of TB. Below are the comments made by participants:

One participant indicated that: "We have a serious need for the good quality equipments and personal measures such as $N 95$ respiratory masks, gloves and protective clothes like that of the theatres to protect us from contracting infection with our clothes and expose our families into risks of contracting infection."

Another participant said: "We need infection control and prevention to be in place for our safety. It is important that after having contact with patients we should wash our hands with soup. Provincial people always say never risk going in the TB wards without protective materials."

The findings of this study revealed that infection control and prevention are not available in TB wards and nurses expressed needs of them. Nurses and patients were exposed at a great risk of contracting TB infection in the wards. The findings of this study are in agreement with the findings of the study that was done Tshitangano et al. (2013) [8], which concludes that infection control plans 
are not available at the hospitals in Vhembe district and this result in high risk of TB infection transmission in the hospitals.

\subsubsection{Sub-Theme 2.3 Special Support from the Management}

Nurses play a vital role in the hospital of caring for patients. They are the main core for the hospital to produce good and quality results. Therefore it is important for the hospital CEO and nursing unit managers to support them in order for them to remain productive. The hospital should make sure that they order quality equipment, and other materials to assist nurses to do their work. There should be provided with adequate and necessary training to empower them with relevant skills and knowledge. Good management empowers employees in the hospital in different ways and also listens to their requests. Below are the comments made by the participants:

One participant said: "Hmm we believe that for now we just need a special support from the management and all TB program managers, to empower us with knowledge maybe by having workshops with us or by arranging in-service training. It is crucial that we gain more knowledge on how to communicate with patients who are showing side effects who are easily to default when they are at home. I believe that if we can be empowered with knowledge death rate and the spread of disease can be reduced. Most patients default because there do not receive support and there is no one do DOT them so we need knowledge to empower patients."

Another participant said: "I believe that it is important to have meetings with doctors and other management to give us in-service training and workshop us."

According to the findings of this study it is revealed that nurses have various unmet needs as they are doing their job. Participants are expressed the needs of special support from the management. They were in need for more adequate supplies of specialized protective N95 mask, in-service training, workshops, proper equipments and daily diet for TB nurses. A study that was done by Sodeify, Vanaki and Mohammadi (2013) [18], revealed that the poor working conditions, lack of resources, special managerial support from the hospitals in South Africa were the reasons why nurses left South Africa to work abroad.

\subsection{Limitations}

The study focused on the support needs of six nurses caring for TB patients at Tshilidzini Hospital in Limpopo Province, South Africa and therefore the findings cannot be generalized. However, generalizability of results in a qualitative study should be treated with caution [21]. The hospital where the study was conducted is situated in the rural area. If other hospitals had been included, it could have led to different data findings.

\section{Conclusion}

According to the findings of this study, it was revealed that nurses are in need of special support from their managers and colleagues. It was also discovered that 
infection-control measures, and safety measures are not available in the hospital. Nurses are in need of personal protective materials, regularly serviced infection control and prevention measures. Nurses are also in need of in-service training and workshops so they can provide quality nursing care to the patients. There is a need of special diet for both nurses and TB patients to boost their immune system. Therefore, there is an urgent need to address this problem by the Department of Health, Hospital Executive Managers and Nursing Service Manager.

\section{Acknowledgements}

I feel a deep sense of gratitude to Dr. A. K. Tugli and Prof. D. U. Ramathuba (UNIVEN), the TB nursing staff at Tshilidzini hospital, and the Department of Health-Limpopo Province for the roles they played in carrying out this study.

\section{Conflicts of Interest}

The authors declare no conflicts of interest regarding the publication of this paper.

\section{References}

[1] Talip, B.A., Sleator, R.D., Lowery, C.J., Dooley, J.S.G. and Snelling, W.J. (2013) An Update on Global Tuberculosis (TB). Infectious Diseases: Research and Treatment, 6, 39-50. https://doi.org/10.4137/IDRT.S11263

[2] World Health Organization (WHO) (2018) Global Tuberculosis Report. WHO, Geneva, Switzerland.

[3] Valjee, L. and van Dyk, A.C. (2014) Impact of Caring for People Living with HIV on the Psychosocial Well-Being of Palliative Caregivers. Curationis, 37, 1-13. https://doi.org/10.4102/curationis.v37i1.1201

[4] Nasreen, S., Shokoohi, M. and Malvankar-Mehta, M.S. (2016) Prevalence of Latent Tuberculosis among Health Care Workers in High Burden Countries: A Systematic Review and Meta-Analysis. PLoS ONE, 11, e0164034. https://doi.org/10.1371/journal.pone.0164034

[5] Statistics South Africa (2017) Mortality and Causes of Death in South Africa: Findings from Death Notification. Stats SA, Pretoria, 2018.

[6] Limpopo Provincial TB Annual Report (2010) TB Annual Report.

[7] Sukumani, J.T., Lebese, R.T., Khoza, L.B. and Risenga, P.R. (2012) Experiences of Family Members Caring for Tuberculosis Patients at Home at Vhembe District of the Limpopo Province. Curationis, 35, 1-8. https://doi.org/10.4102/curationis.v35i1.54

[8] Tshitangano, T.G., Maputle, S.M. and Netshikweta, L.M. (2013) Available of Tuberculosis Infection Control Plans at Rural Hospitals of Vhembe District, Limpopo Province of South Africa. African Journal of Primary Health Care \& Family Medicine, 5, 1-6. https://doi.org/10.4102/phcfm.v5i1.480

[9] Zelnick, J.R., Gibbs, A., Loveday, M., Padayatchi, N. and O’Donnel, M.R. (2013) Healthcare Workers Perspective on Workplace Safety, Infection Control and Drug Resistant Tuberculosis in High-Burden HIV Setting. Journal of Public Health Policy, 34, 388-402. https://doi.org/10.1057/jphp.2013.20

[10] Gursimrat, K.S. (2011) Tuberculosis: Current Situation, Challenges and Overview of 
Its Control Programs in India. Faculty of Medicine, Lund University, Lund, Sweden.

[11] Tshitangano, T.G. (2013) Factors That Contribute to Public Sector Nurses Turnover in Limpopo Province of South Africa. African Journal of Primary Health Care \& Family Medicine, 5, 1-7. https://doi.org/10.4102/phcfm.v5i1.479

[12] Wolmarans, M. and Asia, B. (2013) Limpopo Vhembe District Profile.

[13] Colaizzi, P.F. (1978) Psychological Research as the Phenomenologist Views It. In: Valle, R. and King, M., Eds., Existential Phenomenological Alternatives for Psychologists, Oxford University Press, New York.

[14] Manyisa, Z.M and van Aswegen, E.J. (2017) Factors Affecting Working Conditions in Public Hospitals: A Literature Review. International Journal of Africa Nursing Sciences, 6, 28-38. https://doi.org/10.1016/j.ijans.2017.02.002

[15] Mametja, V.L. (2013) Problem Experienced by Professional Nurses Caring for HIV/AIDS Patients in Public Hospital of Polokwane Municipality, Limpopo Province of South Africa. Faculty of Health Science, University of Limpopo, Limpopo.

[16] Arjun, S.D. (2011) The Experience of Enrolled Nurses Caring for MDR-TB patients in Kwazulu Natal. University of South Africa, South Africa.

[17] Loghmani, L., Borhani, F. and Abbaszadeh, A. (2014) Factors Affecting the NursePatients' Family Communication in Intensive Care Unit of Kerman: A Qualitative Study. Journal of Caring Sciences, 3, 67-82.

[18] Sodeify, R., Vanaki, Z. and Mohammadi, E. (2013) Nurses' Experiences of Perceived Support and Their Contributing Factors: A Qualitative Content Analysis. Iranian Journal of Nursing and Midwifery Research, 18, 191-197.

[19] South Africa (2007) The Draft National Infection Prevention and Control Guidelines for TB, MDRTB and XDRTB. Government Printers, Pretoria.

[20] Narasimhan, P., Wood, J., Maclntyre, C.R. and Mathai, D. (2013) Risk Factors of Tuberculosis. Pulmonary Medicine, 2013, Article ID: 828939.

https://doi.org/10.1155/2013/828939

[21] Smith, B. (2017) Generalizability in Qualitative Research: Misunderstandings, Opportunities and Recommendations for the Sport and Exercise Sciences. Qualitative Research in Sport, Exercise and Health, 10, 137-149. https://doi.org/10.1080/2159676X.2017.1393221 